\title{
The effect of fish oil on two-step tuberculin test in hospitalized patients: A randomized controlled trial
}

\author{
N. Arfa ${ }^{1}, \underline{\text { A. Allami }}{ }^{1}$ \\ ${ }^{1}$ Department of Infectious Diseases, Qazvin University of Medical Sciences, Qazvin, Iran \\ Corresponding Address: Abbas Allami, Department of Infectious Diseases, Bu-Ali Hospital, Qazvin University of \\ Medical Sciences, Qazvin, Iran \\ Tel: +98-28-33379630, Email: allami9@yahoo.com \\ Received: 20 Sep 2017; Accepted: 13 Jan 2018
}

\section{* Abstract}

Background: According to national tuberculosis control guide, two-step tuberculin skin test (TST) should be done in the elderly, if the initial test is negative. However, it raises questions about the usefulness of this approach.

Objective: This study aimed to explore the effects of fish oil supplements on the two-step tuberculin test in hospitalized patients.

Methods: In this randomized controlled clinical trial, 128 patients randomly allocated to control group (receiving placebo, $\mathrm{n}=64$ ) and treatment group (receiving fish oil supplements, $\mathrm{n}=64$ ) during 2016. Fish oil supplement group was treated with $2 \mathrm{~g}$ daily for 4 consecutive days. The outcome was considered a change in 2 sequential TST induration sizes. Significant increase in the size of the secondary induration compared to primary was considered $6 \mathrm{~mm}$ or more.

Findings: There was significant difference between primary and secondary indurations of two groups (higher in treatment group) $(\mathrm{P}=0.04)$. According to the results of analysis of variance and correlation tests, two effective factors were identified: initial induration and residence location $(\mathrm{P}=0.014$ and $\mathrm{P}=0.002$, respectively). In both groups, no clinically significant increase in the size of induration was observed.

Conclusion: It seems that the number of cases considered as infected with tuberculosis does not increase with two- rather than one-step tuberculin skin test. Also, the short-term administration of fish oil supplements does not change this result.

Keywords: Fish oils, Polyunsaturated fatty acids, Dietary supplements, Tuberculin test, Clinical trial Citation: Arfa N, Allami A. The effect of fish oil on two-step tuberculin test in hospitalized patients: A randomized controlled trial. J Qazvin Univ Med Sci 2018; 21 (6): 22-29. 


\title{
اثر روغن ماهى بر آزمون يوستى توبر كولين دو مرحلهاى در بيماران بسترى: يكى كار آزمايى تصادفى كنترل شده
}

\author{
دكتر نرجس ارفع '، دكتر عباس علامى'
}

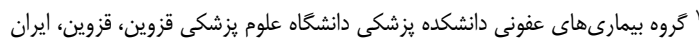

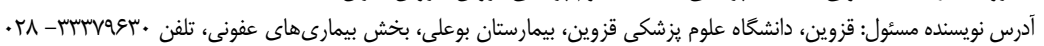

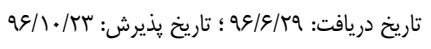

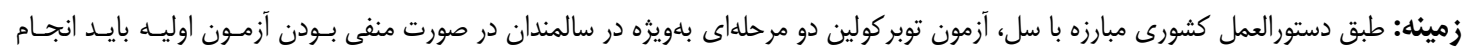

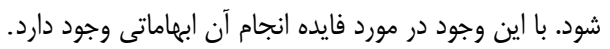

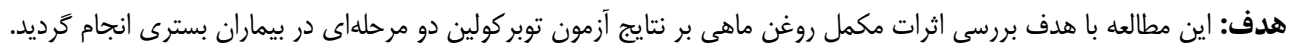

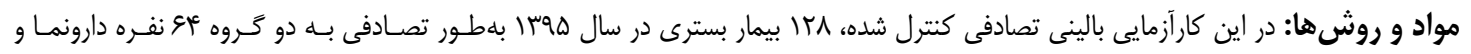

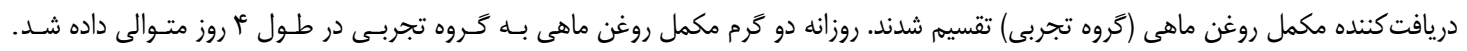

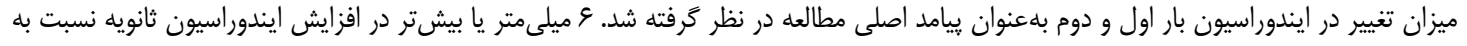

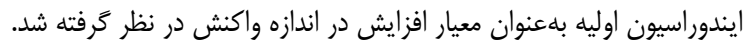

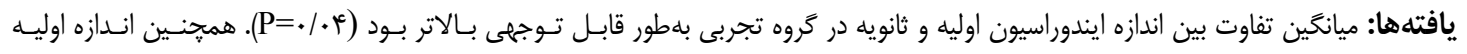

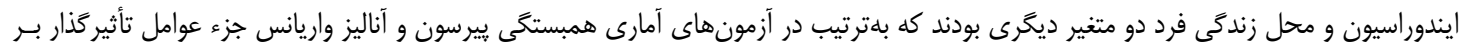

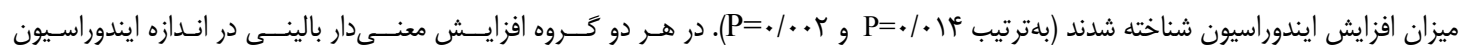
مشاهده نشد.

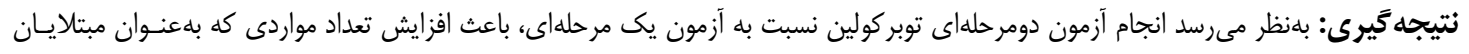

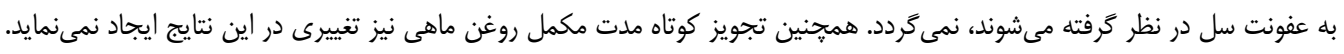

$$
\text { كليدوازهها: روغن ماهى، اسيدهاى جرب اشباع نشده، مكملهاى غذايى، آزمون توبر كولين، كارآزمايى بالينى }
$$

مقدمه:

يا تشخيص ديرهنخـام توسـط يزشـكان) و ورود بيمـاران

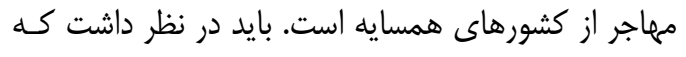

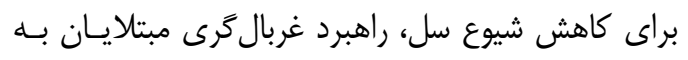

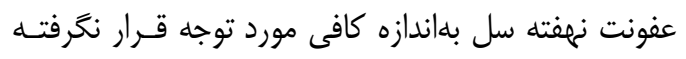

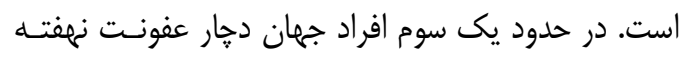

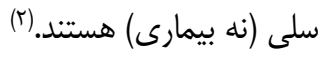

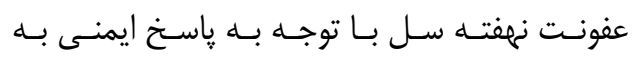

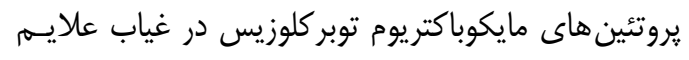

از تعداد ها 99 مورد مبتلا به سل تزارش شده كشـور

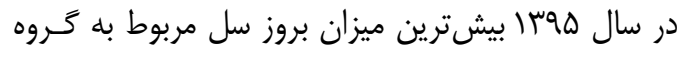

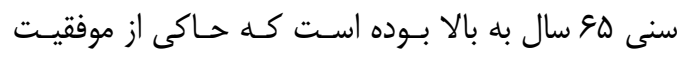

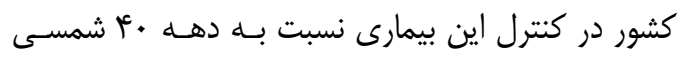

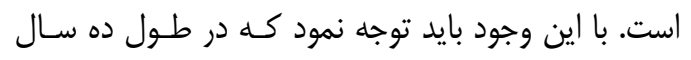

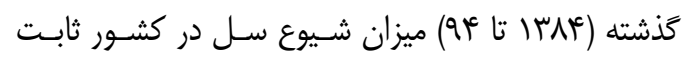

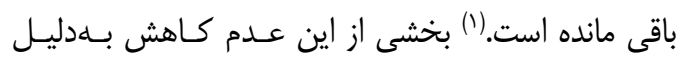

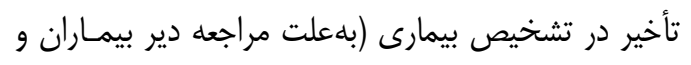




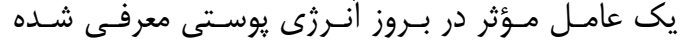

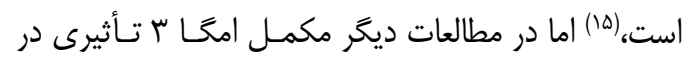

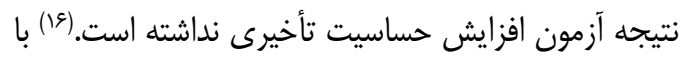

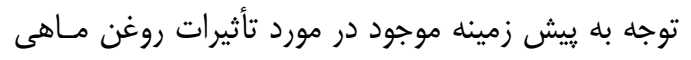

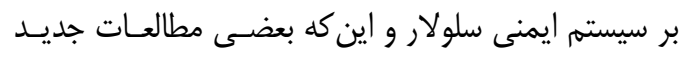

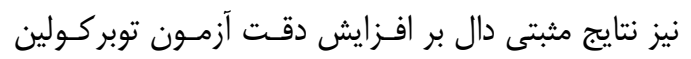

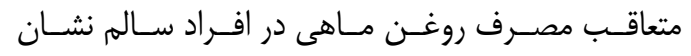

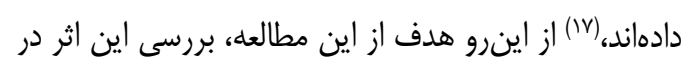
بيماران بسترى در بيمارستان است.

\section{مواد و ورشها:}

اين مطالعه كارآزمايى بالينى تصادفى كنترل شده در

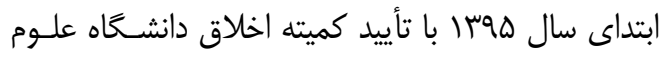

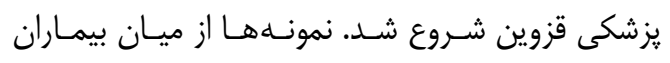
بسترى در بخشىهاى عفـونى، داخلى و اعصـاب مركـز

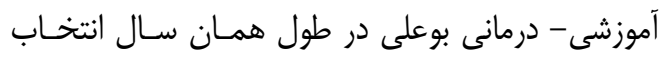

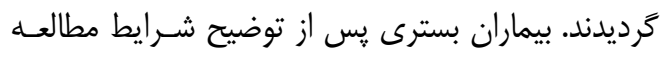

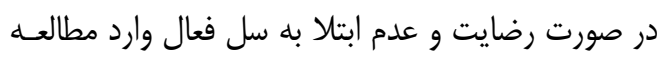

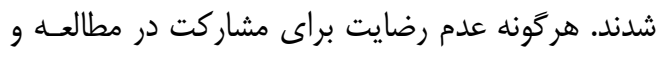

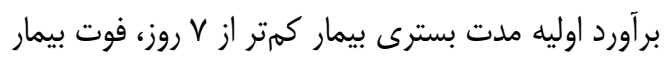

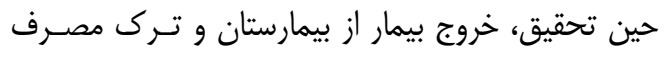
دارو و ترديد در مصرف صحيح دوز تجويز شده بلهعنـوان

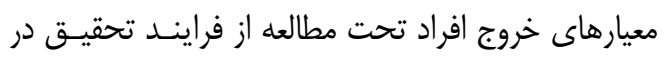

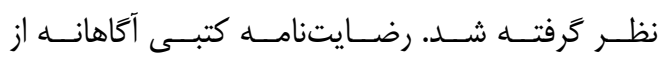

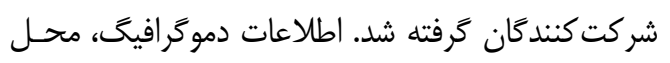

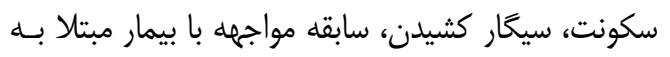

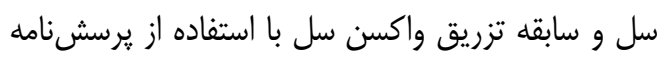
جمع آورى شد. همجنين محل تزريق واكسن بيمـاران از نظر وجود اسكار مورد معاينه قرار كرفت.

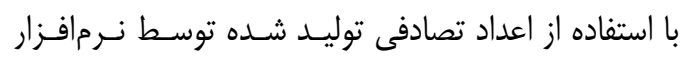
Statics and Sample Size تخصيص تصادفى)، شركت كنندكان بهطور تصادفى در دو

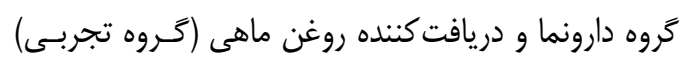

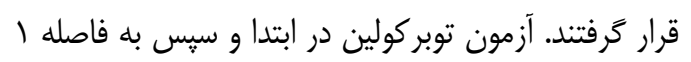

بالينى و نشانههايى از بيمـارىهـاى فعـال تعريـف شـده

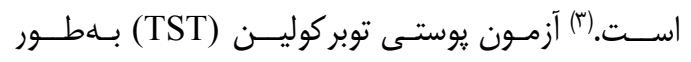

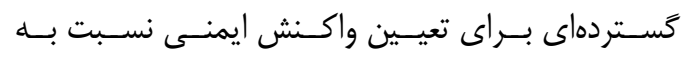

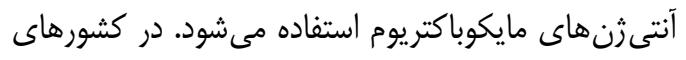
توسعه يافته، براساس نتايج آزمون توبر كولين در مورد نياز

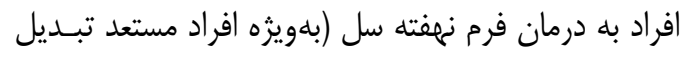

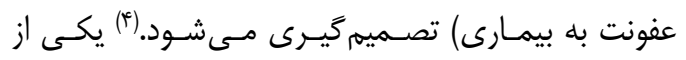

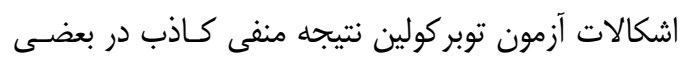

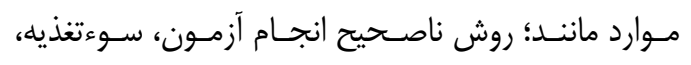

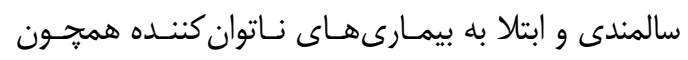

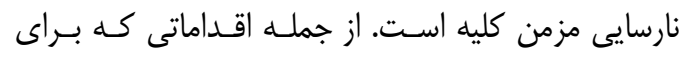

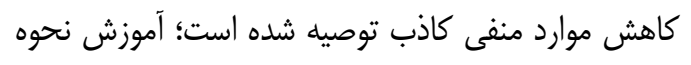

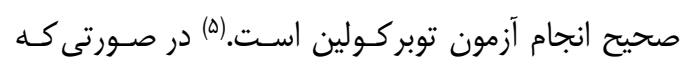

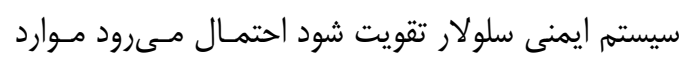

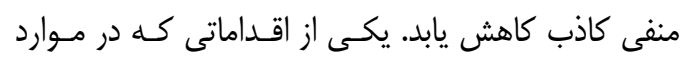

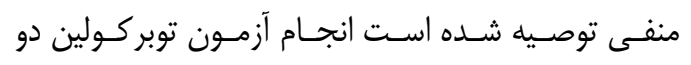

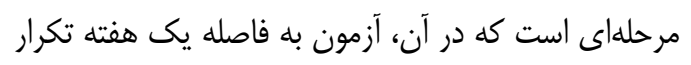

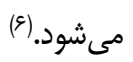
امخا س روغن مـاهى شـامل داكسـاهخزانوئيك اسـيد (DAPA)

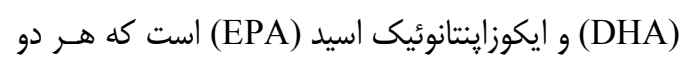

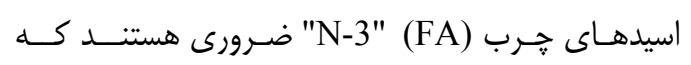

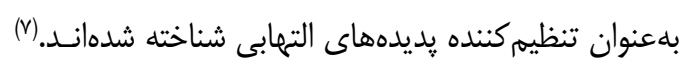

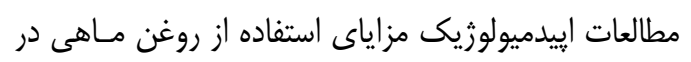

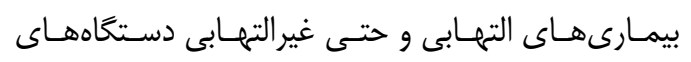

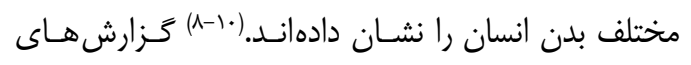

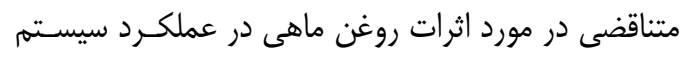

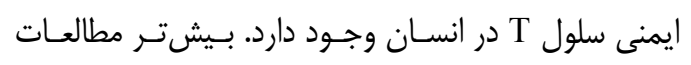

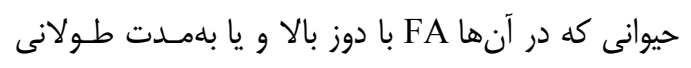

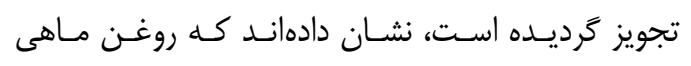

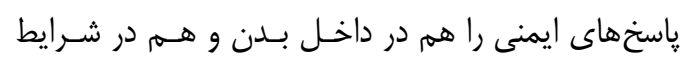

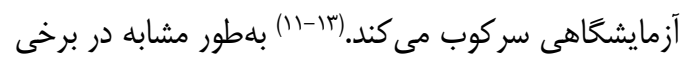

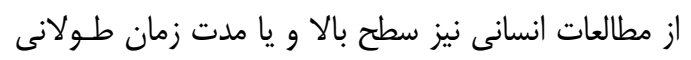

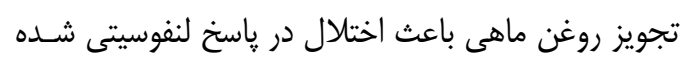

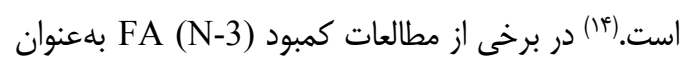


مقدار ه+/P> معنىدار در نظر كرفته شد.

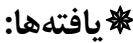

از عٔا نفر كه در ابتداى مطالعه انتخاب شـــه بودنـد،

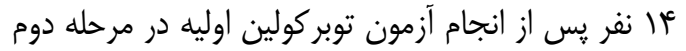

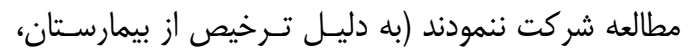

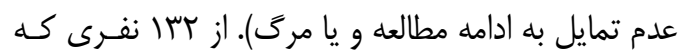

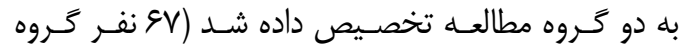

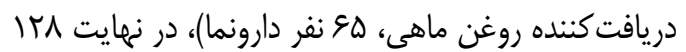

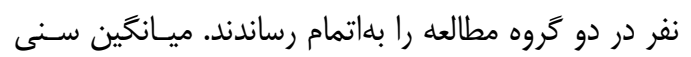

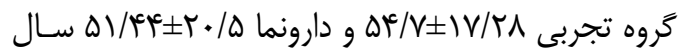

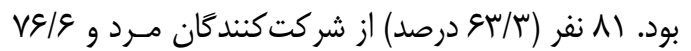

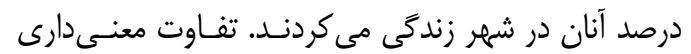

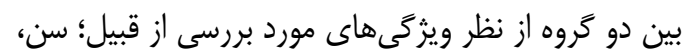

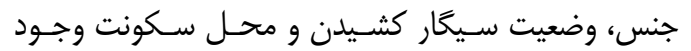

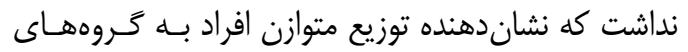

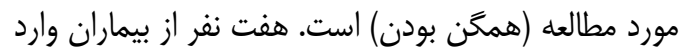
شده بله مطالعه سابقه شناخته شده از تماس با بيمار مبـتلا

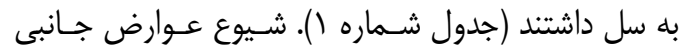
گزارش شده توسط بيماران در دو گروه مشابه يكديخر بود.

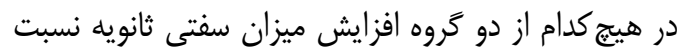

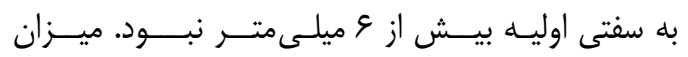

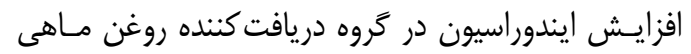

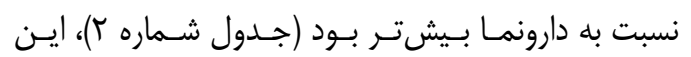

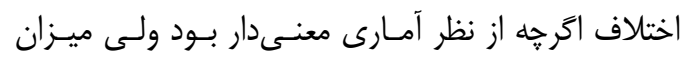

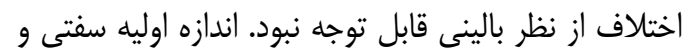

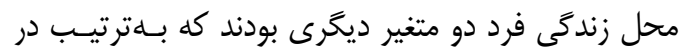

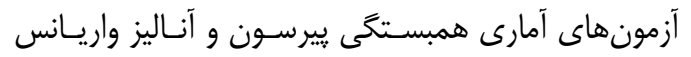

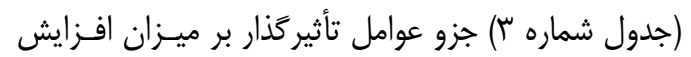

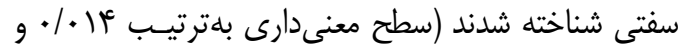

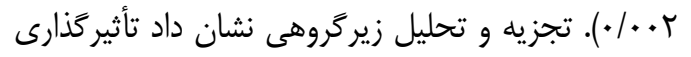

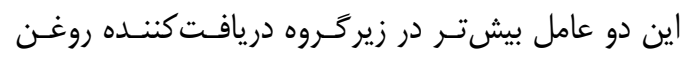
ماهى وجود دارد.
هفته بعد از تلقيح اوليه براى هر دو گروه انجـام مسىشــــ

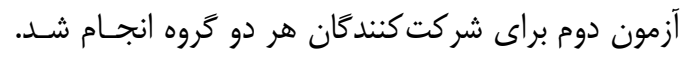

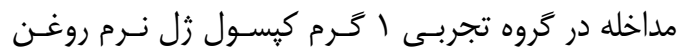

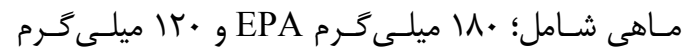
Enriching (Nutralife) EIG توليد شـركت) DHA Global Health

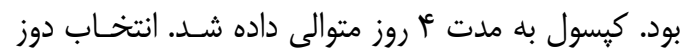

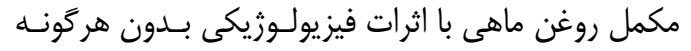

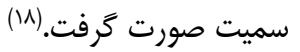
ييامد اصلى تغيير در اندازه سـفتى دو آزمـون متـوالى (اوليه و ثانويه) در نظر كرفته شد. شش ميلى لثمتر يا بيشتر بلهعنوان معيار براى تشـخيص افـزايش ارزشـمند از نظـر

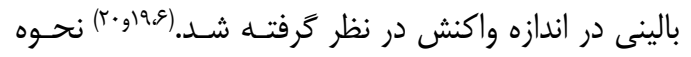

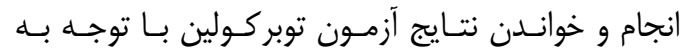
دستور العمل هاى استاندارد به فـــد انجـامدهنــــ (دسـتيار

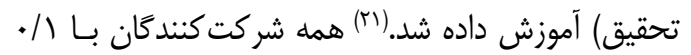

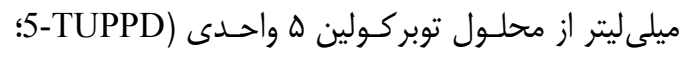

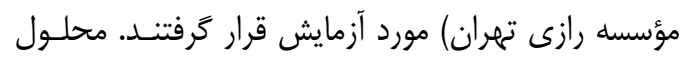

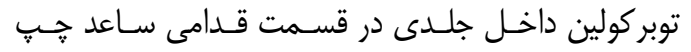

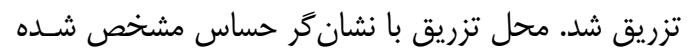

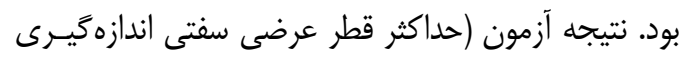

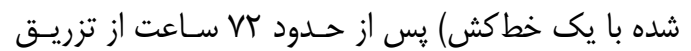

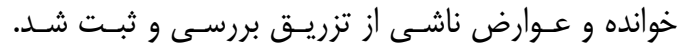
محقق مسئول خواندن نتيجــه دوميـن آزمـون توبر كوليـن به ايـن موضـوع كـه (Tuberculin Skin Test, TST)

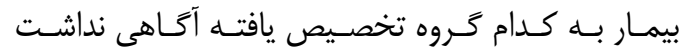

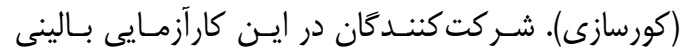
مىتوانستند هر زمان كه بخواهند از مطالعه خارج شوند.

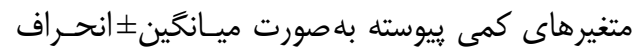
معيار و متغيرهاى كيفى بلهورت فراوانى (درصد) گزارش

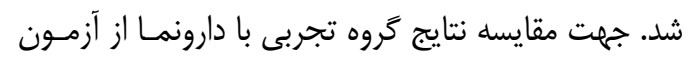

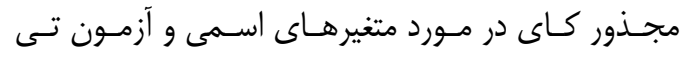

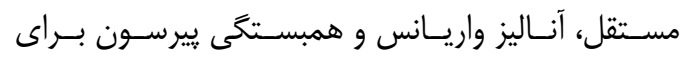

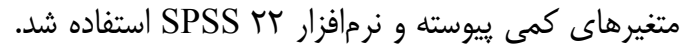




\begin{tabular}{|c|c|c|c|c|c|}
\hline \multirow[t]{2}{*}{ سطح معنى دارى } & \multicolumn{3}{|c|}{ ميانكين+انحراف معيار يا (درصد) تعداد } & \multirow{2}{*}{\multicolumn{2}{|c|}{ متغير }} \\
\hline & مجموع & دارونما & روغن ماهى & & \\
\hline ש זr/. & $\Delta \Psi / \cdot V \pm I N / Q \varepsilon$ & $\Delta T / K \cdot \pm F+/ \Delta$ & $\Delta F / I V \pm V / T \Lambda$ & & سن \\
\hline \multirow{2}{*}{ عس/. } & ᄉ) $(q \mathbb{R} / \Gamma)$ & 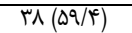 & W ( $(S V / T)$ & مرد & \multirow[b]{2}{*}{ جنس } \\
\hline & FV (re/V) & $\frac{1}{r g(f \cdot / 9)}$ & T) (TY/A) & زن & \\
\hline \multirow{2}{*}{$.1 .9 *$} & u (VG/q) & $\Delta \Gamma(\Lambda T / \Lambda)$ & $F \Delta(V \cdot / \mu)$ & شهر & \multirow{2}{*}{ محل زندگى } \\
\hline & $r \cdot(r M / F)$ & $11(I V / T)$ & $19(\mathrm{rq} / \mathrm{V})$ & روستا & \\
\hline \multirow{3}{*}{. $/$ Ar } & $1 \cdot \Delta(\Lambda T)$ & $\Delta r(\Lambda) / r)$ & $\Delta \omega^{\top}(\Lambda Y / \Lambda)$ & عفونى & \multirow{3}{*}{ بخش بسترى } \\
\hline & $19(I T / \Delta)$ & $9(118 / 1)$ & $V(1 . / 9)$ & داخلى & \\
\hline & $V(\Delta / \Delta)$ & $r(F / v)$ & $f(\xi / \mu)$ & ICU & \\
\hline \multirow{2}{*}{.$/ 4 V$} & $T /(1 \& / 4)$ & $9(15 / 1)$ & $\pi(\Lambda / \Lambda)$ & دارد & \multirow{2}{*}{ استعمال سيگار } \\
\hline & $1 \cdot V(\Lambda \mu / \mathcal{L})$ & $\Delta \Delta(\Lambda \Delta / ৭)$ & $\Delta r(\Lambda) / \widetilde{T})$ & ندارد & \\
\hline \multirow{2}{*}{$\cdot / \Lambda \Delta$} & $\Lambda \Delta(\xi q / f)$ & fr $(g \Delta / 9)$ & $F^{N}(g V / T)$ & دارد & \multirow{2}{*}{ اسكار } \\
\hline & 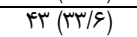 & $r t(M Y / F)$ & T) (Tr/A) & ن ندارد & \\
\hline \multirow{3}{*}{$\cdot 1 \cdot V^{*}$} & Vq $(g, / v)$ & 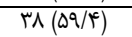 & f) $(94 / 1)$ & ن ن ندارم & \multirow{3}{*}{ تماس با بيمار سل } \\
\hline & $\nabla(\Delta / \Delta)$ & $T(1 / 9)$ & $9(9 / 4)$ & دارم & \\
\hline & FT (Tr/A) & $r \Delta(r q / \backslash)$ & $\operatorname{IV}(r \varepsilon / \mathcal{G})$ & خاطر ندارم & \\
\hline \multirow{3}{*}{$\cdot / 4 V$} & $I(\cdot / \Lambda)$ & $1(1 / 9)$ & $\cdot$ & 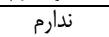 & \multirow{3}{*}{ سابقه واكسن سل } \\
\hline & $\wedge(\varepsilon / \mu)$ & $r(f / V)$ & $\Delta(V / \Lambda)$ & دارم & \\
\hline & $119(94)$ & $9 \cdot(94 / \Lambda)$ & $\Delta q(9 T / T)$ & خاطر ندارم & \\
\hline
\end{tabular}

جدول r- مقايسه متغيرهاى بيامد و عوارض در طى دو مر حله به تفكيك تروه تجربى و دارونما

\begin{tabular}{|c|c|c|c|c|}
\hline \multirow[t]{2}{*}{ سطح معنىدارى } & \multicolumn{3}{|c|}{ ميانكين ثانحراف معيار يا (درصد) تعداد } & \multirow[t]{2}{*}{ متغير } \\
\hline & مجموع & دارونما & روغن ماهى & \\
\hline \multicolumn{5}{|r|}{ اندازه ايندوراسيون } \\
\hline$\cdot|\wedge|$ & $1 / \Delta \pm r / \Delta \varphi$ & $1 / F T \pm r / \Delta D$ & $1 / \Delta \wedge \pm r / \Delta q$ & اوليه \\
\hline.$/ 48$ & I/Af $\pm r / q)$ & $1 / \Delta \wedge \pm r / q q$ & $r / \cdot q \pm r / I r$ & ثانويه \\
\hline$\cdot 1 \cdot 4^{2}$ & $\cdot / \mu f \pm 1 / \cdot 1$ & $\cdot / 19 \pm \cdot / V T$ & $\cdot / \Delta Y \pm I / T Y$ & ميزان اختلاف \\
\hline \multicolumn{5}{|r|}{ عوارض دارويى } \\
\hline \multirow{4}{*}{.$/ 91$} & $1.9(\Lambda \Delta / T)$ & $\Delta f(\Lambda F / F)$ & $\Delta \Delta(\wedge \Delta / \mathrm{Q})$ & ندارد \\
\hline & $\Delta(r / q)$ & $r(r / V)$ & $r(r / J)$ & تهوع \\
\hline & $1 \cdot(V / \Lambda)$ & $\Delta(V / \Lambda)$ & $\Delta(V / \Lambda)$ & مزه \\
\hline & $f(r / l)$ & $r(r / I)$ & $r(r / I)$ & نفخ \\
\hline
\end{tabular}

جدول بـ مقايسه ميزان اختلاف اندازه ايندور اسيون به تفكيك تروهها و متغيرهاى كيفى (تجز يه و تحليل زيرتحروهى)

\begin{tabular}{|c|c|c|c|c|}
\hline \multirow[t]{2}{*}{ سطح معنى دارى } & \multicolumn{2}{|c|}{ (انحراف معيار) سايز ايندوراسيون } & \multirow{2}{*}{\multicolumn{2}{|c|}{ متغير }} \\
\hline & دارونما & روغن ماهى & & \\
\hline \multirow{2}{*}{. $/ 4 T \mid$} & $\cdot / Y \backslash(\cdot / \wedge \mathrm{V})$ & •/ & مرد & \multirow{2}{*}{ جنس } \\
\hline & $\cdot / \cdot \wedge(\cdot /$ १q $)$ & $\cdot / T r(\cdot / 9 V)$ & زن & \\
\hline \multirow{2}{*}{$\cdot 1+\cdot r^{*}$} & $\cdot 1 \cdot 9(\cdot / 4 \cdot)$ & $\cdot / 4 q(\cdot / \wedge q)$ & شهر & \multirow{2}{*}{ محل زندگى } \\
\hline & $\cdot / \& \Delta(1 / \Delta I)$ & $1 / \cdot \Delta(\backslash / \& \Lambda)$ & روستا & \\
\hline \multirow{3}{*}{.$/ 9.9$} &.$/ 19(\cdot / 199)$ & $\cdot / \Delta Q(\backslash / K \Psi)$ & عفونى & \multirow{3}{*}{ بخش بسترى } \\
\hline & $\cdot$ & $\cdot / \Delta \mathrm{V}(\mathrm{I} / \Delta \mathrm{\Delta})$ & داخلى & \\
\hline & $\cdot$ & • & ICU & \\
\hline \multirow{2}{*}{$\cdot / 1 \cdot r$} & $\cdot / I T(\cdot / V T)$ & $\cdot / \mathbb{E}(\backslash / / T)$ & ندارد & \multirow{2}{*}{ استعمال سيگار } \\
\hline & $\cdot / K(\cdot / V I)$ &.$/ 9 Y(1 / D 1)$ & دارد & \\
\hline \multirow{2}{*}{ • } & $\cdot / \cdot 9\left(\cdot /\left.\right|^{4}\right)$ & $\cdot / \mu \Psi(1 / \cdot 9)$ & ندارد & \multirow{2}{*}{ اسكار } \\
\hline & $\cdot / 19(\cdot / \wedge r)$ & $\cdot / 8 \cdot(1 / 49)$ & دارد & \\
\hline \multirow{3}{*}{$.1 .4 \Lambda^{*}$} & $\cdot / 4)(\cdot / \Lambda \mathrm{V})$ & 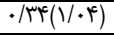 & 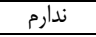 & \multirow{3}{*}{ تماس با بيمار سل } \\
\hline & $\cdot$ & $1 / \Delta \cdot(1 / V 9)$ & دارم & \\
\hline & $\cdot / \cdot \wedge(\cdot / \mathrm{H} \cdot)$ & $\cdot / \Delta q(1 / \mu r)$ & خاطر ندارم & \\
\hline \multirow{3}{*}{.$/ F V T$} & $\cdot$ & $\cdot$ & ندارم & \multirow{3}{*}{ سابقه واكسن سل } \\
\hline & $\cdot$ & $1 / 4 \cdot\left(1 / 9 T^{4}\right)$ & دارم & \\
\hline & $\cdot / I V\left(\cdot / V V^{c}\right)$ & $\cdot / f \&(\backslash / 1 \Lambda)$ & خاطر ندارم & \\
\hline
\end{tabular}




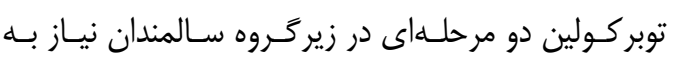
بازنخرى دارد.

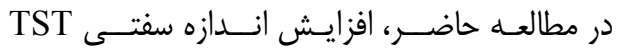

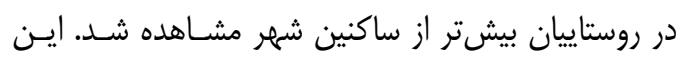

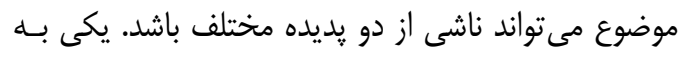

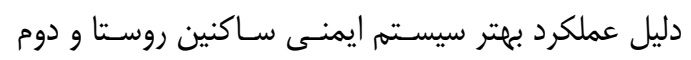

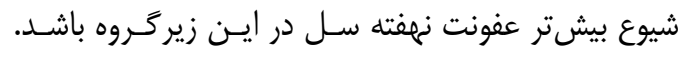

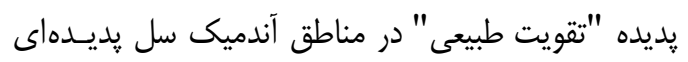

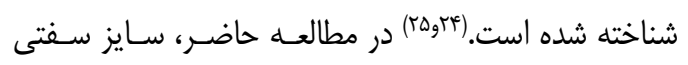

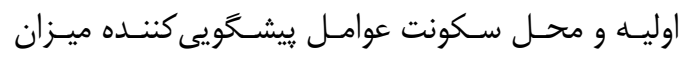

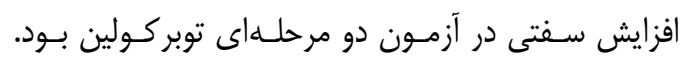

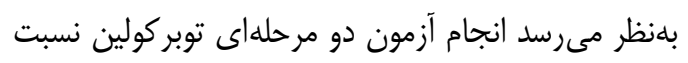

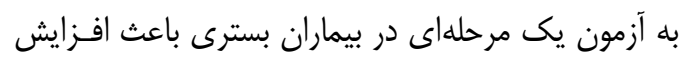

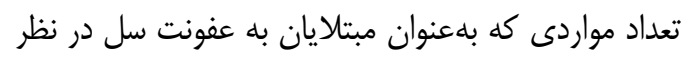

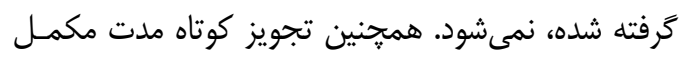

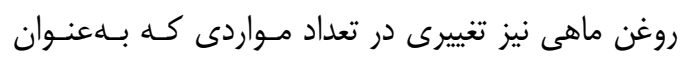
مبتلايان به عفونت سل در نظر كرفتـه مسى نــود، ايجـاد نمىنمايد. از آنجا كه در اين مطالعـه يـــ جمعيـت محسـدود از بيماران در يكى مركز آموزشى -درمانى مورد مطالعه قـرار

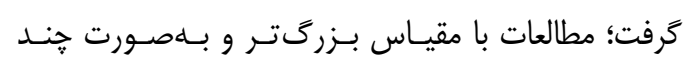

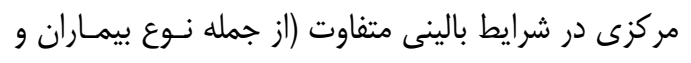
دوز تجويزى روغن ماهى) براى تأييد اين نتيجهَّيرى نياز

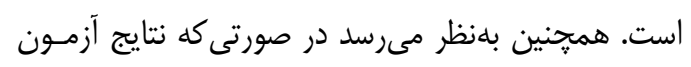

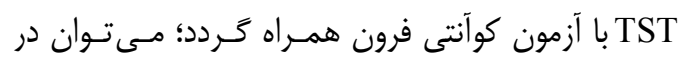

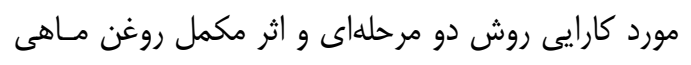

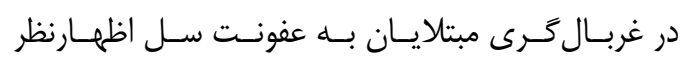
دقيقترى نمود.

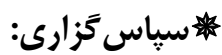

بدينوسيله از حمايـت معاونـت تحقيقـات و فنـاورى

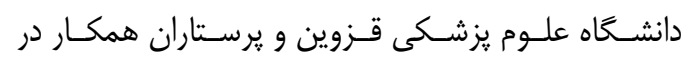

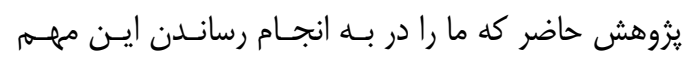

\section{مبحث و نتيجه كيرى}

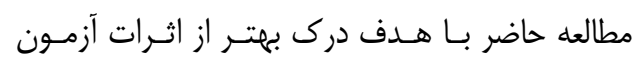

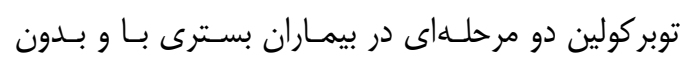

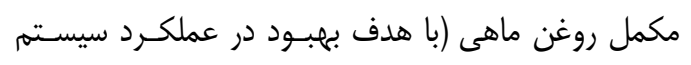

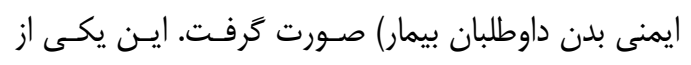

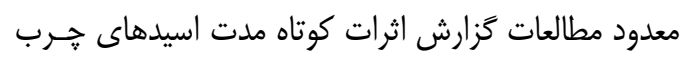

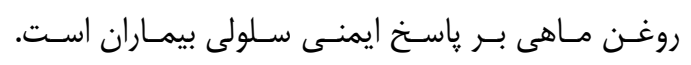

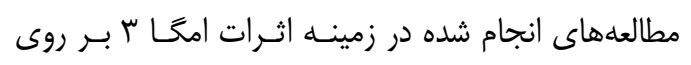

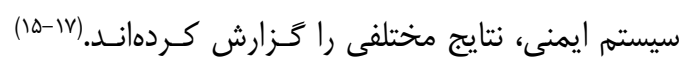

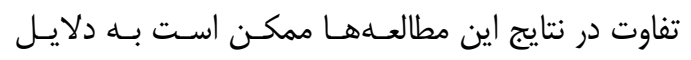

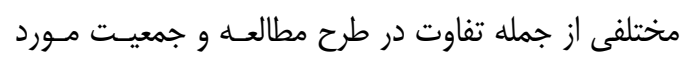
بررسى اين مطالعهها باشد. در مطالعه حاضر، افزايش ميزان سفتى ثانويـه نسـبت إندا.

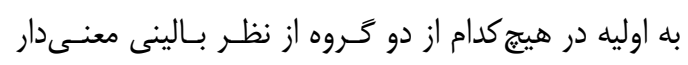

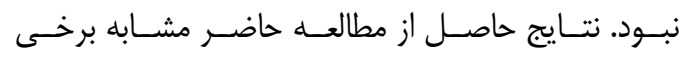
مطالعههاى كذشته مى باشد. در مطالعه مورتى و همكاران

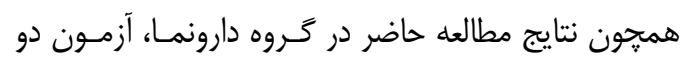

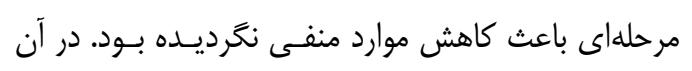

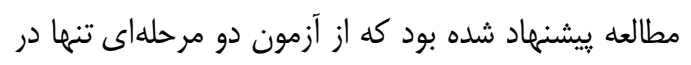

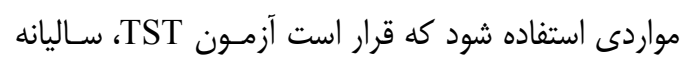

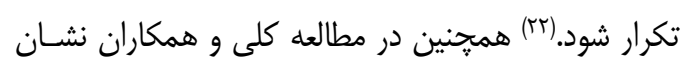

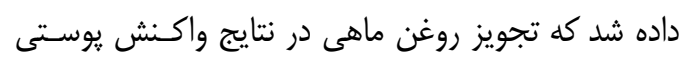

افزايش حساسيت تأخيرى، تغييرى ايجاد نمى نمايد. (T) دادي

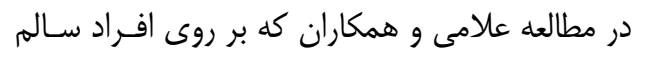

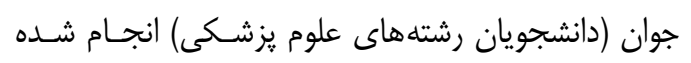

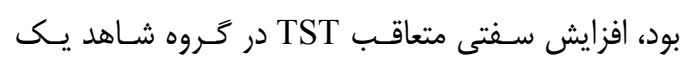

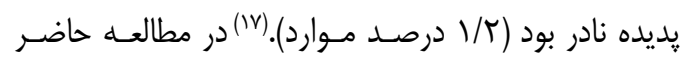

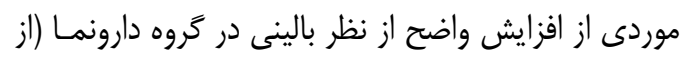

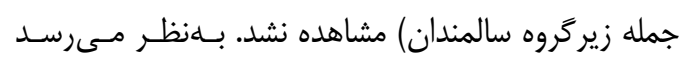

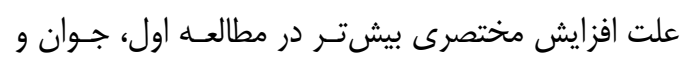

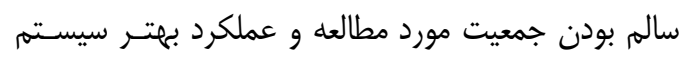

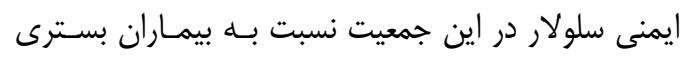

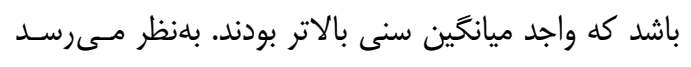

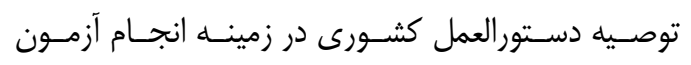


7. Miles EA, Calder PC. Influence of marine n-3 polyunsaturated fatty acids on immune function and a systematic review of their effects on clinical outcomes in rheumatoid arthritis. Br J Nutr 2012; 107(Suppl 2): S171S84. doi: 10.1017/S0007114512001560.

8. Bazinet RP, Layé S. Polyunsaturated fatty acids and their metabolites in brain function and disease. Nat Rev Neurosci 2014; 15(12): 771-85. doi: 10.1038/nrn3820.

9. Calder PC. Omega-3 polyunsaturated fatty acids and inflammatory processes: nutrition or pharmacology? Br J Clin Pharmacol 2013; 75(3): 645-62. doi: 10.1111/j.1365-2125. 2012.04374.x.

10. Mickleborough TD. Omega-3 polyunsaturated fatty acids in physical performance optimization. Int $\mathbf{J}$ Sport Nutr Exerc Metab 2013; 23(1): 83-96.

11. de Arruda LLM, Ames FQ, de Morais DR, Grespan R, Gil APM, Silva MARCP, et al. A single administration of fish oil inhibits the acute inflammatory response in rats. Asian Pac J Trop Med 2017; 10(8): 765-72. doi: 10.1016/j.apjtm.2017.07.019.

12. Dasilva G, Pazos M, García-Egido E, Gallardo JM, Rodríguez I, Cela R, et al. Healthy effect of different proportions of marine $\omega-3$ PUFAs EPA and DHA supplementation in Wistar rats: Lipidomic biomarkers of oxidative stress and inflammation. J Nutr Biochem 2015; 26(11): 1385-92. doi: 10.1016/j.jnutbio.2015.07.007. 13. Gibson D, Gill SK, Brown K, Tasnim N, Ghosh S, Innis S, et al. Maternal exposure to fish oil primes offspring to harbor intestinal pathobionts associated with altered immune cell balance. Gut Microbes 2015; 6(1): 24-32. doi: 10.1080/19490976.2014.997610.

14. Simopoulos AP. Omega-3 fatty acids in inflammation and autoimmune diseases. J Am

$$
\begin{aligned}
& \text { يارى نمودند، تشكر و قدردانى مىنماييمه. }
\end{aligned}
$$

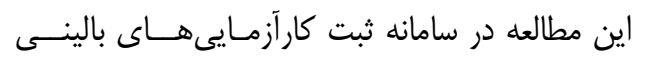

$$
\begin{aligned}
& \text { ايسران (IRCT) به شماره IRCT2015070520882N4) } \\
& \text { ثبت كرديده است. } \\
& \text { مراجع: }
\end{aligned}
$$

1. Estimates of TB and MDR-TB burden are produced by World Health Organization in consultation with countries 2016. Generated: 2018-02-27, Available at: https://extranet.who.int/sree/Reports?op=Repl et\&name=/WHO_HQ_Reports/G2/PROD/EX $\mathrm{T} / \mathrm{TBC}$ countryProfile\&ISO2=IR\&outtype $=\mathrm{ht}$ $\mathrm{ml}$ [cited $2018 \mathrm{Feb} 27$ ].

2. WHO. Tuberculosis 2016. Available from:http://www.who.int/mediacentre/factshe ets/fs104/en/. Updated in: 2017 Mar.

3. Barry CE 3rd, Boshoff HI, Dartois V, Dick T, Ehrt S, Flynn J, et al. The spectrum of latent tuberculosis: rethinking the biology and intervention strategies. Nat Rev Microbiol 2009; 7(12): 845-55. doi: 10.1038/ nrmicro2236.

4. Sheldon L. Morris. Developing a Clinical Tuberculin Test: Tuberculin Characteristics. In: Reichman LB, Bhavaraju R, editors. Guidelines for the Diagnosis of Latent Tuberculosis Infection in the 21st Century. 2nd ed. Newark: New Jersey Medical School Global Tuberculosis Institute; 2008. 18-23.

5. Nasehi M, Mirhaghani L. National tuberculosis control guide. 2nd ed.Tehran: Andishmand Publisher; 2009. 5-21. [In Persian].

6. Menzies D. Interpretation of repeated tuberculin tests: boosting, conversion, and reversion. Am J Respir Crit Care Med 1999; 159(1): 15-21. doi: 10.1164/ajrccm.159.1. 9801120. 
Coll Nutr 2002; 21(6): 495-505.

15. D'vaz N, Meldrum SJ, Dunstan JA, Lee-Pullen TF, Metcalfe J, Holt BJ, et al. Fish oil supplementation in early infancy modulates developing infant immune responses. Clin Exp Allergy 2012; 42(8): 1206-16. doi: 10.1111/j.1365-2222.2012. 04031.x.

16. Song HJ, Grant I, Rotondo D, Mohede I, Sattar N, Heys SD, et al. Effect of CLA supplementation on immune function in young healthy volunteers. Eur J Clin Nutr 2005; 59(4): 508-17. doi: 10.1038/sj.ejen. 1602102.

17. Allami A, Haji Ali F, Jafarpour H, Mohammadi N. The effect of fish oil fatty acid supplementation on two-step tuberculin skin test: a randomized controlled clinical trial. Biotech Health Sci 2017; 4(1): e39876. doi: 10.5812/bhs. 39876 .

18. Simopoulos AP. Omega-3 fatty acids and cardiovascular disease: The epidemiological evidence. Environ Health Prev Med 2002; 6(4): 203-9. doi: 10.1007/BF02897971.

19. Menzies R. Interpreting repeated tuberculin skin tests. In: Reichman LB, editors. Guidelines for the diagnosis of latent tuberculosis infection in the 21st century. 2nd ed. Newark: New Jersey Medical School Global Tuberculosis Institute; 2008. 38-46.

20. Monárrez-Espino J, Enciso-Moreno JA, Laflamme L, Serrano CJ. Serial QuantiFERON-TB Gold In-Tube assay and tuberculin skin test to diagnose latent tuberculosis in household Mexican contacts: conversion and reversion rates and associated factors using conventional and borderline zone definitions. Mem Inst Oswaldo Cruz 2014; 109(7): 863-70. doi: 10.1590/00740276140085
21. U.S. Department of Health and Human Services Centers for Disease Control and Prevention NCfHA, Viral Hepatitis, STD, and TB Prevention. Latent tuberculosis infection: a guide for primary health care providers, appendix C: Administration and measurement of the TST 2013. Available at: http://www.cdc.gov/tb/publications/LTBI/app endixC.htm. Updated in: April 3, 2013 [cited 2018 Feb 27].

22. Murthy M, Selvam S, Jesuraj N, Bennett S, Doherty M, Grewal HM, et al. Two-step tuberculin skin testing in school-going adolescents with initial $0-4$ millimeter responses in a high tuberculosis prevalence setting in South India. PloS One 2013; 8(9): e71470. doi: 10.1371/journal.pone.0071470.

23. Kelley DS, Taylor PC, Nelson GJ, Mackey BE. Dietary docosahexaenoic acid and immunocompetence in young healthy men. Lipids 1998; 33(6): 559-66.

24. Schiller I, Vordermeier HM, Waters WR, Whelan AO, Coad M, Gormley E, et al. Bovine tuberculosis: effect of the tuberculin skin test on in vitro interferon gamma responses. Vet Immunol Immunopathol 2010; 136(1-2): 1-11. doi: 10.1016/j.vetimm.2010. 02.007 .

25. Beveridge NE, Price DA, Casazza JP, Pathan AA, Sander CR, Asher TE, et al. Immunisation with $\mathrm{BCG}$ and recombinant MVA85A induces long-lasting, polyfunctional Mycobacterium tuberculosisspecific CD4+ memory $\mathrm{T}$ lymphocyte populations. Eur J Immunol 2007; 37(11): 3089-100. doi: 10.1002/eji.200737504 